\title{
Effects of gluten enriched diet on the small intestinal mucosa of normal mice and mice with graft versus host reaction
}

\author{
R Troncone, N Caputo, A Zibella, G Molitierno, L Maiuri, S Auricchio
}

\begin{abstract}
This study looked at the effect of extra dietary gluten on the intestinal architecture of both normal mice and those with an ongoing mucosal delayed hypersensitivity reaction. BDF1 normal mice and mice in which a graft $v$ host reaction (GvHR) had been induced, both weaned on gluten free diet, were allocated for three weeks to three different dietary regimens: gluten free, 'normal' (3.6\% gluten), and gluten enriched $(15 \cdot 8 \%$ gluten). In normal mice receiving the gluten containing diet, shorter villi, deeper crypts, and higher crypt cell production rate were noted when compared with those receiving gluten free diet: these changes were more pronounced in those receiving the gluten enriched diet. GvHR mice showed shorter villi and an increase in both crypt length and crypt cell production rate when compared with normal mice, but the presence of gluten in their diet did not produce additional damage. Both in normal and in GvHR mice receiving gluten containing diet there were no signs of systemic (cell mediated or humoral) or mucosal immune reactions (raised intraepithelial lymphocyte counts or enhanced epithelial Ia expression) to gliadin. In conclusion, increasing the dietary gluten content produces significant changes in the mucosal architecture of normal mice; mice with GvHR enteropathy do not show additional damage resulting from dietary gluten.
\end{abstract}

(Gut 1994; 35: 779-782)

Gluten is an important constituent of our diet. The average daily gluten intake per person is comparatively constant, but, whereas this amount is normally harmless, it is clearly sufficient to induce dramatic changes of the jejunal mucosal architecture in genetically susceptible subjects. Most evidence suggests an immune mediated hypersensitivity reaction as the most probable mechanism of this enteropathy. ${ }^{1}$

There is also evidence from published works, however, of a dose related gluten sensitive enteropathy. While considerable amounts of gluten given to military volunteers produced no symptoms or lesions of the intestinal mucosa, ${ }^{2}$ some patients with dermatitis herpetiformis, ${ }^{34}$ some first degree relatives of coeliac patients, ${ }^{5}$ and even some normal subjects ${ }^{4}$ respond to gluten loading with diarrhoea and show jejunal biopsy abnormalities. It has been suggested that the worsening of the mucosal changes occurs only in those subjects who have latent gluten sensitivity. Nevertheless it is still unclear if this phenomenon is entirely a result of immunological reaction, or whether other factors related to the physicochemical nature of gluten contribute to the mucosal damage.

The aim of our work was to test, in an animal model, the effect of a gluten enriched diet on the small intestinal mucosa. Both normal mice and mice with an ongoing mucosal delayed type hypersensitivity reaction were investigated. In the second group we tested the hypothesis that the mucosa could be more sensitive to gluten induced damage in a condition characterised by accelerated epithelial turnover.

\section{Methods}

ANIMALS AND DIETS

Animals - (C57BL/6J x DBA2) F1 (H/2 b/d) (BDF1) female mice were used (Charles River Italia, Calco, Como, Italy). They were weaned on a gluten free diet and were maintained under conventional conditions before use in the study at the age of $6-8$ weeks. Each experimental group consisted of six to eight mice.

Diets - three different diets with identical protein, fat, cellulose, and ashes content (18, 4,6 , and $6.5 \%$, respectively) were used (Mucedola, Settimo Milanese, Italy). The gluten free diet was prepared with potato flour, peanut powder, dry non-fat milk, and soy oil. The 'normal' gluten containing diet was a standard rodent diet, gluten contributing about $20 \%$ to the total protein content, with maize, fish, soy, milk whey accounting for the remaining $80 \%$. The gluten enriched diet was similar, with the exception of the protein component, to which gluten contributed $88 \%$ and milk whey protein the remaining $12 \%$. All diets were equally supplemented with vitamins and oligoelements; in particular, the content of iron, manganese, zinc, copper, and cobalt was identical. The calculated daily gluten intake per mouse for the three diets was none, 150 $\mathrm{mg}$, and $750 \mathrm{mg}$ respectively.

Graft versus host reaction - a graft versus host reaction was induced by the intraperitoneal injection of $10^{8} \mathrm{C} 57 \mathrm{BL} 6 / \mathrm{N}$ spleen cells into 
BDF1 mice. Spleen cell suspensions were made with RPMI 1640 medium (Gibco), with viability assessed by $2 \%$ trypan blue exclusion as greater than $90 \%$. At the time of their death, animal body and spleen weights were measured and Simonsen's spleen index calculated. ${ }^{6}$ A spleen index of $>2$ was considered to be significant.

\section{INTESTINAL MORPHOMETRY}

Crypt cell production rate - all groups of animals were given $7.5 \mathrm{mg}$ colchicine (Sigma) per kg bodyweight and killed by cervical dislocation at intervals of 20-120 minutes afterwards, to permit accumulation of metaphases in intestinal crypts. Pieces of jejunum were fixed in $75 \%$ ethanol $/ 25 \%$ glacial acetic acid and stained in bulk with the modified Feulgen stain (Schiff reagent, Difco). For each animal, accumulated metaphases were counted in at least 20 crypts. The crypt cell production rate was expressed as the rate of accumulation of metaphases/crypt/hour.

Villous/crypt dimensions - dimensions of villi and crypts were also measured by the microdissection techniques. ${ }^{7}$ Using an eyepiece graticule in a light microscope, the height of villi and the depth of crypts were measured. Fifteen measurements of villus and crypt length were made in each specimen and the mean calculated.

Intraepithelial lymphocyte counts - pieces of jejunum were fixed in $10 \%$ buffered formalin, embedded in paraffin wax, cut at $5 \mu \mathrm{m}$, and stained with haematoxylin and eosin. A differential count was made under oil immersion ( $\times 1000$ magnification) of the cells within the epithelium covering the villi, and the intraepithelial lymphocyte counts expressed as number of intraepithelial lymphocyte per 100 epithelial cells.

\section{ASSESSMENT OF THE IMMUNE STATE}

Antigliadin antibodies - mice were bled from the retro-orbital plexus under light ether anaesthesia and the serum tested for IgG antibodies to gliadin by an enzyme linked immunosorbent assay (ELISA) technique. Microtitre plates (Flow Laboratories) were coated by overnight incubation at room temperature with a solution containing $1 \mu \mathrm{g} / \mathrm{ml}$ of gliadin (kindly provided by $\mathrm{Dr} \mathrm{H}$ Wieser, Deutsche Forschungsanstalt, Garching, Germany) in $70 \%$ ethanol. Test samples were diluted 1:1000 with phosphate buffered saline containing $10 \%$ horse serum (Seromed, Germany) (PBS-HS). These were added to the plate, which was then incubated for two hours at room temperature. Affinity purified antimouse IgG conjugated with horseradish

TABLE I Body weights of normal and GvHR BDF1 mice on three diets with different gluten content

\begin{tabular}{llll}
\hline & Gluten free & Normal & Gluten enriched \\
\hline Normal mice & $22 \cdot 6(1 \cdot 1)(n=7)$ & $23 \cdot 2(1 \cdot 7)(n=7)$ & $23 \cdot 5(1 \cdot 2)(n=7)$ \\
GvHR mice & $16 \cdot 5(4 \cdot 5)(n=4)$ & $19 \cdot 4(3 \cdot 3)(n=7)$ & $21 \cdot 1(3 \cdot 0)(n=7)$ \\
\hline
\end{tabular}

Values in grams, mean (SD). peroxidase (Sigma) was added at 1:200 dilution and the plates incubated for a further two hours at room temperature. O-phenylenediamine (Merck) at concentration $1 \mathrm{mg} / \mathrm{ml}$ in phosphate-citrate buffer $\mathrm{pH} 5.0$ was used as substrate, and the absorbance read at $450 \mathrm{~nm}$ using an automatic ELISA reader (Titertek Multiskan, Flow). Between each incubation step the plates were washed three times with PBS containing 0.05\% TWEEN 20 (Sigma).

Epithelial expression of class II antigens - to investigate the epithelial expression of Ia antigens, pieces of jejunum were frozen in isopentane fluid cooled in liquid nitrogen and $5 \mu$ cryostat tissue sections were air dried and then fixed for 10 minutes in acetone. Staining was performed using a mouse (IgG2a) monoclonal antibody directed at a highly conserved antigenic determinant expressed on most murine class II antigens. ${ }^{8}$ (gift of $M$ Pierres, INSERM, Marseille Luminy, France), and a fluorescein isothiocyanate goat antimouse $\mathrm{Ig}$ (Becton-Dickinson, Mountain View, CA, USA). The sections were incubated with $3 \%$ normal goat serum to prevent non-specific binding. Histochemical specificity tests included: (a) omission of the primary antibody, (b) substitution of the primary antibody with a mouse monoclonal antibody with different specificity but of the same isotype.

Specific delayed type hypersensitivity - mice were also tested for systemic delayed type hypersensitivity to gliadin. Footpad thickness was measured by skinfold calipers (Mitutoyo, Japan), immediately before and 24 hours after an intradermal injection of $25 \mu \mathrm{g}$ of gliadin in $0.05 \mathrm{ml}$ of water.

\section{STATISTICS}

Student's $t$ test was used to compare villous and crypt lengths and intraepithelial lymphocyte counts. Crypt cell production rate was calculated from the least squares estimate of the linear regression of number of blocked metaphases with time after colchicine injection. Crypt cell production rates were compared by covariance analysis.

\section{Results}

General - no differences were recorded between mice belonging to the three different dietary regimens, gluten free, 'normal', and gluten enriched, as far as body weights (Table I) and serum total protein content were concerned. Mice undergoing GvHR weighed (mean (SD)) significantly less than controls $(19.0(3.3)$ g $v 23.1(1.4) ; \mathrm{p}<0.001)$, with no differences between the three dietary groups (Table I); by day 21, 6 of 24 had died (four in the gluten free diet, one in the normal gluten containing diet, one in the gluten enriched diet group).

Effect of gluten enriched diet on the mucosal architecture of normal mice - at the age of 6-8 weeks two groups of BDF1 mice were transferred to a 'normal' gluten containing diet, or to a gluten enriched diet; a third group, which served as control, was maintained on gluten 


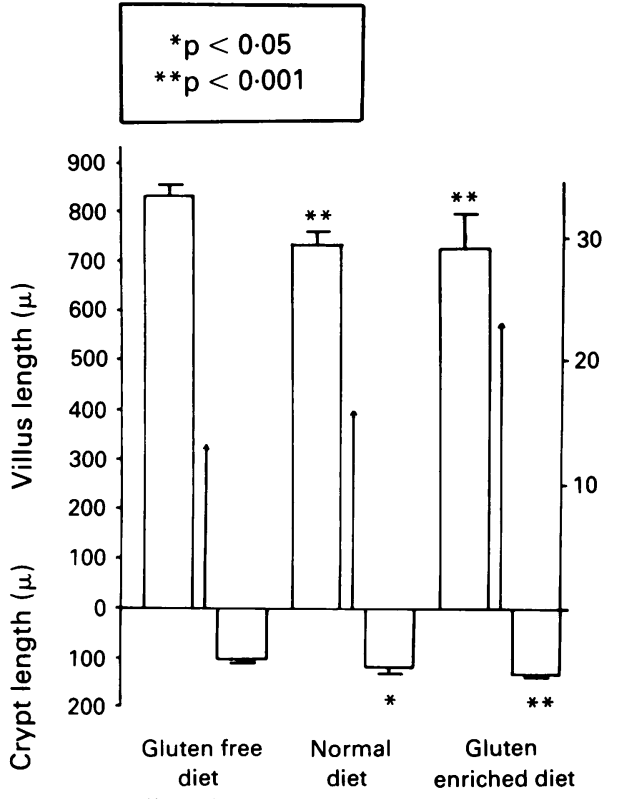

Figure 1: Effect of a gluten enriched diet on the mucosal architecture of normal mice. At the age of 6-8 weeks two groups of gluten free $B D F 1$ mice were transferred to a 'normal' gluten containing diet, or to a gluten enriched diet, a third group, that served as control, was maintained on gluten free diet. Mice were killed three weeks afterwards, and jejunal samples obtained for a morphometric evaluation. A mild but significant shortening of villi was seen, as well as increasing values of crypt length and crypt cell production rate (CCPR) (shown by the arrow), with the increasing content of gluten in the diet.

free diet. Mice were killed three weeks afterwards, and samples from the proximal jejunum obtained for a morphometric evaluation. Mild but significant villous shortening was seen in mice on gluten containing diet, with increased crypt length and crypt cell production rate found with increasing dietary gluten content. These results were consistently obtained in three different experiments; in Figure 1 the results of a representative experiment are shown.

Effects of gluten enriched diet on the mucosal architecture of mice undergoing GvHR - when the jejuna of normal mice and mice with GvHR on gluten free diet were compared three weeks after the induction of the reaction, a significant shortening of villi (640 (29) $v 831$ (22) $\mu ; \mathrm{p}<0.001)$ was seen, together with a marked crypt hyperplasia (130 (21) v 106 (4); $\mathrm{p}<0.05)$. Crypt cell production rate was also increased $(14.0 \vee 13 \cdot 3)$, although the difference was not statistically significant. To test the effect of additional dietary gluten on the mucosal architecture of GvHR mice, two groups of such mice were transferred to a normal gluten containing diet or a gluten enriched diet for three weeks, a third group on gluten free diet serving as control. When the three groups of GvHR mice, on the three different dietary regimens were compared, no significant differences in villus and crypt

TABLE II Intraepithelial lymphocyte (IEL) counts in normal and GvHR BDF1 mice on three diets with different gluten content

\begin{tabular}{lrrr}
\hline & Gluten free & Normal & Gluten enriched \\
\hline Normal mice & $12.9(3 \cdot 3)(n=7)$ & $13 \cdot 6(1 \cdot 9)(n=7)$ & $11 \cdot 7(1 \cdot 6)(n=7)$ \\
GvHR mice & $7 \cdot 0(1 \cdot 9)(n=4)$ & $9 \cdot 7(3.4)(n=7)$ & $10 \cdot 6(3 \cdot 4)(n=7)$ \\
\hline
\end{tabular}

IELs/100 epithelial cells, mean (SD).

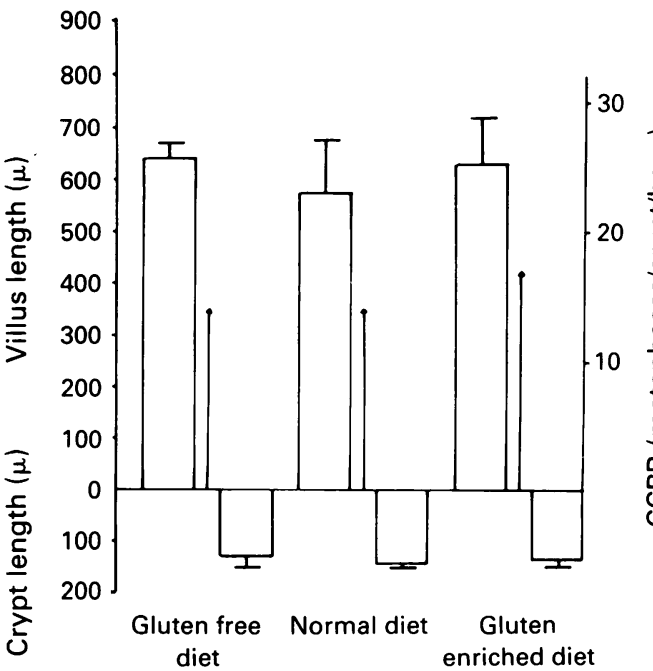

Figure 2: Effects of gluten enriched diet on the mucosal architecture of mice undergoing graft versus host reaction. To test the effect of additional dietary gluten on the mucosal architecture of GvHR mice, two groups of these mice were transferred, at the time of induction of the reaction, to either a normal diet or a gluten enriched diet for three weeks, with a third group on gluten free diet serving as control. No significant differences were evident when villus length, crypt depth, and crypt cell production rate (CCPR) (shown by the arrow) were measured.

length and crypt cell production rate were evident (Fig 2).

Immune response to gliadin in mice on gluten enriched diet - normal mice maintained on gluten enriched diet showed no signs of increased systemic or mucosal immune response. In fact no gliadin antibodies were measurable in the serum and no significant response could be elicited in mice skin tested with gliadin, in comparison with controls skin tested with water. Furthermore, no signs of activated cell mediated mucosal immunity were evident in those mice fed high amounts of gluten; intraepithelial lymphocyte counts were not statistically different in the three dietary groups (Table II). Moreover, Ia expression was not enhanced: in normal gluten free mice crypt enterocytes were negative for Ia. Mice killed after three weeks of gluten enriched diet showed a pattern comparable with these control mice (Fig 3A), in contrast with GvHR mice in which Ia expression was detectable in the crypts (Fig 3B).

\section{Discussion}

In genetically susceptible subjects, such as coeliac patients or patients with dermatitis herpetiformis, gluten is responsible for mucosal changes in the jejunum. It is now increasingly recognised that the spectrum of such lesions is much wider than previously thought, the mucosa progressing in a sequential manner through several phases (infiltrative, hyperplastic, and destructive), before becoming flat. ${ }^{1}$ The increased amount of dietary gluten is probably responsible for this evolution.

The mechanisms for this dose dependent gluten sensitive enteropathy are still not completely understood. One possibility is that the damage is entirely due to a hypersensitivity reaction. An alternative possibility is related to 
Figure 3: Ia staining in crypt enterocytes of normal mice on gluten enriched die (A), and of GvHR mice (B). (A) In mice on gluten enriched diet crypt enterocytes do not show Ia molecule expression, the pattern being similar to that of mice on gluten free dict: (B) in GvHR mice, which represent a 'positive' control of activated mucosal cell mediated immunity, Ia expression is significantly enhanced in the cytoplasm of the enterocytes of some crypts (original magnification $\times 100)$.
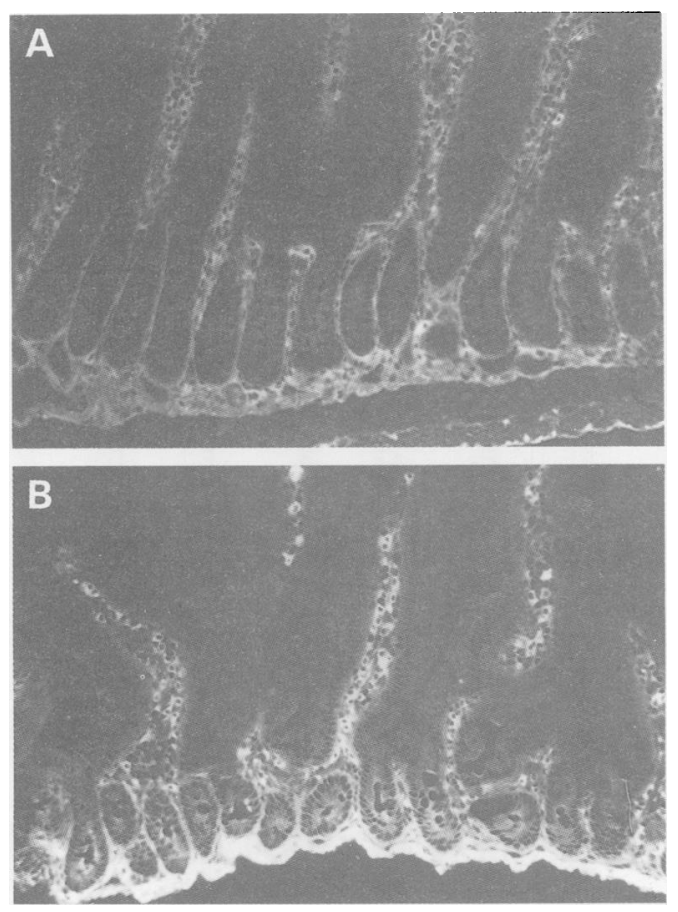

the very peculiar physicochemical structure of gliadin, which has a tendency to interact with immature cell structures. ${ }^{9}$ In a condition of accelerated cell turnover, such as that caused by an ongoing mucosal cell mediated immune reaction, gliadin could contribute to the damage because of these properties.

Different authors have studied the effect of wheat proteins on the jejunal mucosa of rodents. ${ }^{1011}$ The results of these studies are difficult to compare, because of differences in the nature of the protein used (wheat proteins, gluten or gliadin), in the duration of challenge (single dose or few days challenge), in study protocol (sensitisation by previous treatment) or in the assessment of damage (permeability or gross morphological changes). In this study we aimed at assessing, in an animal model, the longterm effect of increased dietary gluten on jejunal architecture. We found a dose dependent lesion characterised by shortening of villi, crypt hyperplasia, and increased crypt cell production rate. This finding differs from that in our earlier report, ${ }^{12}$ in which we failed to show any gluten induced change in the jejunum of unprimed mice from a gluten free colony: in that case, however, the duration of gluten challenge was shorter and the amount of daily dietary gluten less.

The mechanism by which such changes have been induced is still unclear. We have no definite evidence in favour of an immune mediated reaction, either at systemic level (there were no signs of specific humoral or cell mediated response), or within the mucosa (intraepithelial lymphocyte counts did not increase, nor was there enhanced expression of Ia molecules), Nevertheless, it is difficult to exclude with certainty a low grade immune response to an antigen never encountered before. An alternative possibility is that of direct cytotoxic activity. This effect has been shown in a number of models including agglutination of undifferentiated K562 cells and inhibition of in vitro differentiation of fetal rat intestine: all such data suggest the propensity of gliadin peptides to interact with immature cell structures. For this reason we have tested the hypothesis that a gluten enriched diet would be even more active on less mature enterocytes, such as those found in a mucosa undergoing a delayed type hypersensitivity reaction, which is characterised by increased epithelial cell turnover. Using murine graft versus host reaction, in which by three weeks there is already mucosal damage characterised by crypt hyperplasia, villous shortening, and depletion of intraepithelial lymphocytes, ${ }^{13}$ we could not induce any additional damage by increasing dietary gluten: the two insults do not seem to be additive. This is possibly explained by the severity of the changes already induced by the graft versus host reaction or by the difficulty of inducing villous atrophy in mice, or both.

In conclusion, increasing the dietary gluten content produces significant changes in the mucosal architecture of normal mice. Mice with GvHR enteropathy, however, do not show additional damage resulting from dietary gluten. Further studies are needed to establish why increasing dietary gluten induces pathological changes in mice (and some humans), although, at least in the case of this animal model, there is no evidence that immunological mechanisms play a significant part.

The technical assistance of Maria Cucciardi and Giuseppina Riccio is gratefully acknowledged. We are indebted to Dr H Wieser for providing us with gliadin and to Dr M Pierres for supplying monoclonal antibodies to murine MHC class II antigens. We are also grateful to Dr Simon Murch for revising the manuscript. This work has been supported with grants from Regione Campania (Interventi nel settore delle malattic dell (n) favore dei malati affetti da fibrosi cistica).

This study has been presented at the 25 th Annual Mecting of the European Society for Paediatric Gastroenterology and Nutrition (ESPGAN) held in Brussels, 27-30 May 1992, and published in abstract form.

1 Marsh MN. Gluten, major histocompatibility complex, and the small intestine. A molecular and immunobiological approach to the spectrum of gluten sensitivity ("celiac sprue'). Gastroenterology 1992; 102: 330-54.

2 Levine RA, Briggs GW, Harding RS, Nolte LB. Prolonged gluten administration in normal subjects. $N$ Engl f Med 1966; 274: 1109-14

3 Weinstein WM. Latent celiac spruc. Gastrocnterologv 1974; 66. 489-93.

4 Ferguson A, Blackwell J, Barnetson R. Effects of additional dietary gluten on the small-intestinal mucosa of voluntecrs and of patients with dermatitis herpetiformis. Scand 7 Gastroenterol 1987; 22: 543-9.

5 Doherty M, Barry RE. Gluten-induced mucosal changes in subjects without overt small-bowel disease. Lancet 1981; ; 517-20.

6 Simonsen M. Graft versus host reaction. Their natural history and applicability as tools of research. Progress in Allergy 1962; 6: 349-476.

7 Clarke RM. Mucosal architecture and epithelial cell production rate in small intestine of the albino rat. 7 Allu 1970; 107: 519-29.

8 Pierres M, Lafuse WP, Dosseto M, Devaux C, Birnbaum DZ, David CS. Molecular specificity of a monoclonal anti-Ik alloantibody identifying a highly conserved determinant on mouse I-A, I-E and human DR antigens. Teterminant on mouse 1-A, 1-E and

9 Auricchio $S$, Troncone R. In vitro methods for the study of celiac disease. In: Braski D, Rozen P, Kagnoff MF, eds.
Gluten-sensitive enteropathy. Frontiers of Gastrointestinal Gluten-sensitive enteropathy. Frontiers
Research. Basle: Karger, 1992: 44-51.

10 Williams EW, Wodd HP. The effect of wheat proteins on rat intestinal villi. IRCS Med Sic 1981; 9: 23-4

1 Sandhu J, Fraser DR. Effect of dietary cereals on intestinal permeability in experimental enteropathy in rats. Gu 1983; 24: 825-30.

12 Troncone R, Ferguson A. Animal model of gluten-induced enteropathy in mice. Gut 1991; 32: 871-5.

13 Mowat AMcI, Felstein MV. Experimental studies of immunologically mediated enteropathy. V. Destructive enteropathy during an acute graft-versus-host reaction in adult BDF1 mice. Clin Exp Immunol 1990; 79: 279-84. 